\title{
Descripción de un brote epidémico de malaria en una comunidad indígena asentada en la zona urbana de Armenia, Colombia, 2012
}

\author{
Liliana Quintero', Magda Beatriz López¹, Harold Ramírez¹, Jhon Carlos Castaño² \\ 1 Secretaría de Salud de Armenia, Armenia, Colombia \\ 2 Grupo de Inmunología Molecular (GYMOL), Universidad del Quindío, Armenia, Colombia
}

Introducción. La malaria o paludismo es la enfermedad parasitaria transmitida por mosquitos vectores más frecuente en las regiones tropicales. Debido a diversos factores, incluido el cambio climático, la enfermedad puede aparecer o reaparecer en diferentes lugares del mundo.

Objetivo. Describir un brote autóctono de malaria urbana en la ciudad de Armenia, Colombia.

Materiales y métodos. Después de la consulta en un hospital de segundo nivel del sur de Armenia de dos niños con síntomas de malaria, cuyo diagnóstico mediante gota gruesa confirmó la presencia de trofozoítos de Plasmodium vivax, se visitó el sector donde residían para la búsqueda activa de casos. Se describieron las condiciones ambientales y socioculturales del sector y se recolectaron e identificaron entomológicamente larvas, pupas y adultos de mosquitos. Igualmente, se implementaron medidas de control y se evaluó su efectividad.

Resultados. La búsqueda activa en la comunidad permitió la identificación de 11 casos probables de malaria, de los cuales se confirmaron por microscopía tres casos de malaria por $P$. vivax en niños. El análisis entomológico de las larvas y pupas de mosquitos mostró la presencia de Anopheles spp., Anopheles punctimacula y Anopheles argyritarsis.

Conclusiones. Se reportó el primer brote autóctono de malaria por P.vivax en Armenia, asociado con un asentamiento subnormal en una comunidad indígena y se constató cómo en los márgenes del río Quindío se presentan las condiciones eco-epidemiológicas que permiten el ciclo de reproducción del mosquito vector de la malaria.

Palabras clave: malaria, epidemias, población indígena, Plasmodium vivax, Anopheles.

doi: http://dx.doi.org/10.7705/biomedica.v35i1.2195

Outbreak of malaria in an indigenous population living in an urban area of Armenia, Colombia, 2012

Introduction: Malaria is the most frequent mosquito-borne parasitic infection in the tropical regions of the world. Due to different factors, including climate change, this disease can emerge or reemerge in different areas in the planet.

Objective: To describe an autochthonous outbreak of urban malaria in Armenia, Colombia.

Materials and methods: After two children consulted a second level hospital located in the south of Armenia with malaria symptoms and their diagnosis was confirmed through the presence of Plasmodium vivax trophozoites detected by a positive thick blood smear, a visit was conducted to the residence of the children. An active search for cases was carried out, the environmental and sociocultural conditions were described and mosquito larvae and pupae were collected and identified. Control measures were implemented and their effectiveness was evaluated as well.

Results: The active search in the community allowed the identification of 11 probable cases, three of which were $P$. vivax malaria cases in children confirmed by blood microscopy. The entomological analysis of mosquito larvae and pupae showed Anopheles spp., Anopheles punctimacula and Anopheles argyritarsis.

Conclusion: This is the first report of an autochthonous malaria outbreak by $P$. vivax in the city of Armenia, associated with the subnormal settlement of an indigenous community. The banks of the Quindío river offer the eco-epidemiological conditions that allow the establishment of the reproductive cycle of the malaria mosquito vector.

Key words: Malaria, epidemics, Indigenous population, Plasmodium vivax, Anopheles.

doi: http://dx.doi.org/10.7705/biomedica.v35i1.2195

\section{Contribución de los autores:}

Liliana Quintero: asesoría de la revisión, corrección del manuscrito e ideas para la discusión

Jhon Carlos Castaño: revisión de la literatura científica y redacción del manuscrito

Magda Beatriz López y Harold Ramírez: participación en la fase de campo y en la elaboración del informe de estas actividades que sirvió de base para la redacción del manuscrito 
La malaria, o paludismo, es una infección parasitaria causada por protozoarios del género Plasmodium y transmitida al humano por mosquitos vectores. Su presencia es muy frecuente en las regiones tropicales del mundo, donde 3.300 millones de personas de 106 países viven en áreas de riesgo para la transmisión de la enfermedad y entre 300 y 500 millones se infectan anualmente con Plasmodium spp. La malaria es responsable de 1,5 a 2,7 millones de muertes al año, principalmente en niños. La Organización Mundial de la Salud (OMS) estimó que en el 2010 se presentaron 219 millones de episodios clínicos y 660.000 muertes por malaria (1-3).

En Colombia, la transmisión de la malaria es un grave problema de salud pública, ya que cerca de $85 \%$ del territorio rural colombiano está situado por debajo de los 1.600 metros sobre el nivel del mar y presenta condiciones climáticas, geográficas y epidemiológicas aptas para la transmisión de la enfermedad. Se estima que entre 12 y 25 millones de personas habitan en zonas de riesgo para la transmisión de la malaria, la cual se considera una de las enfermedades infecciosas más importantes en el país. Si bien la mortalidad por esta enfermedad ha disminuido, la morbilidad se mantiene elevada, con 120.000 a 150.000 casos anuales $(4,5)$. Después de Brasil, Colombia es el segundo país de Suramérica en cuanto al número anual de casos reportados de malaria (6). En el 2013, Aristizábal, et al., reportaron entre el 2010 y el 2012 la situación de malaria en los 45 municipios priorizados en el proyecto "Malaria Colombia" y encontraron que Plasmodium vivax y Plasmodium falciparum fueron las especies causantes de infección más frecuentes; $P$. vivax aportó entre 60 y $70 \%$ de los casos en todo el país y $P$. falciparum, el resto de los casos (7).

La malaria es causada por el protozoo parásito del género Plasmodium, del cual existen seis especies que infectan a los humanos: $P$. vivax, $P$. falciparum, $P$. malariae, $P$. ovale, $P$. knowlesi y $P$. cynomolgy. Estas se transmiten al hombre mediante la picadura de la hembra del mosquito del

\footnotetext{
Correspondencia:

Jhon Carlos Castaño, Grupo de Inmunología Molecular (GYMOL), Facultad de Ciencias de la Salud, Universidad del Quindío, Carrera 15 Calle 12 Norte, Armenia, Colombia Teléfono: (576) 7359392

jhoncarlos@uniquindio.edu.co

Recibido: 08/11/13; aceptado: 10/09/14
}

género Anopheles infectada con esporozoítos del parásito. Vale la pena aclarar que $P$. knowlesi y $P$. cynomolgy no infectan exclusivamente al hombre y tienen carácter zoonótico (8).

En el país se han registrado 43 especies de Anopheles, de las cuales tres se consideran los vectores principales de Plasmodium: Anopheles albimanus, Anopheles nuñeztovari y Anopheles darlingi. Se han considerado, también, cuatro especies como vectores secundarios: Anopheles punctimacula, Anopheles pseudopuctipennis, Anopheles neivaiy Anopheles lepidotus (8). En 1997, Olano, et al., reportaron un aumento del número de casos de malaria urbana en Buenaventura y observaron la presencia de formas inmaduras de An. albimanus y An. nuñeztovari en excavaciones de minería, lagos y estanques para piscicultura localizados en la zona periurbana del municipio (9). En el 2006, Ochoa, et al., publicaron un estudio de la epidemiología de la malaria urbana en Quibdó, capital del Chocó, fruto de un estudio descriptivo de corte transversal llevado a cabo durante cuatro meses, en el cual incluyeron a todos los pacientes diagnosticados con malaria mediante el examen de gota gruesa, independientemente del tipo de Plasmodium encontrado. Los autores resaltaron el alto porcentaje de malaria urbana autóctona (24,4\% causado por $P$. falciparum y $39,1 \%$, por $P$. vivax), asociada con focos periurbanos caracterizados por ser asentamientos subnormales en los que se carecía de una infraestructura urbana de servicios públicos y recolección de basuras, así como por la presencia de vegetación boscosa (10).

El departamento del Quindío, localizado entre los $04^{\circ} 04^{\prime} 41^{\prime \prime}$ y $04^{\circ} 43^{\prime} 18^{\prime \prime}$ de latitud norte y entre los $75^{\circ} 23^{\prime} 41^{\prime \prime}$ y $75^{\circ} 53^{\prime} 56^{\prime \prime}$ de longitud oeste en la región centro-occidente de Colombia, sobre la Cordillera Central, tiene $1.845 \mathrm{~km}^{2}$ de montañas con alturas entre los 900 y los 4.750 metros sobre el nivel del mar; su capital, Armenia, se encuentra a $4^{\circ} 32^{\prime}$ de latitud norte y a $75^{\circ} 41^{\prime}$ de longitud oeste, 290 kilómetros al oeste de Bogotá, en tanto que su altura es de $1.483 \mathrm{msnm}$ y su temperatura promedio está entre 18 y $22^{\circ} \mathrm{C}(11)$.

Según el boletín epidemiológico de la Secretaría de Salud de Armenia, en el 2008 se reportó una incidencia de malaria por $P$. vivax de 19,25 casos importados por 100.000 habitantes, de malaria por $P$. falciparum, de 2,1 casos importados por 100.000 habitantes, y de malaria mixta, de 0,7 casos importados por 100.000 habitantes (10). Según datos de la Secretaría de Salud de Armenia, en el 2010 se 
presentaron 53 casos importados de malaria por $P$. vivax, uno de malaria mixta y 11 de malaria por $P$. falciparum, y en el 2011, 12 casos de malaria importada, 10 casos de malaria por $P$. vivax, uno de infección mixta y otro por $P$. falciparum (12).

El propósito del presente trabajo fue describir un brote de malaria autóctono en una comunidad indígena asentada en la zona urbana de Armenia, Quindío.

\section{Materiales y métodos}

\section{Descripción y control del brote}

Se acogieron las definiciones de 'caso probable' y 'caso confirmado' contenidas en el protocolo de vigilancia y control de la malaria del Instituto Nacional de Salud de Colombia. Caso probable: persona con fiebre actual o reciente procedente de área endémica en los últimos 15 días y que puede tener o no relación epidemiológica (nexo) con casos diagnosticados. Caso confirmado: paciente con episodio febril $\left(>37,5^{\circ} \mathrm{C}\right)$ actual o reciente (hasta en las dos semanas previas) y procedente de área endémica de malaria en los últimos 15 días, cuya enfermedad se confirme por la identificación de especies de Plasmodium mediante examen parasitológico (13).

\section{Descripción de casos índice}

El 12 de diciembre de 2012, mediante fichas de notificación enviadas por el Hospital del Sur, se reportaron dos casos de malaria mixta $(P$. vivax y $P$. falciparum) en un niño de 9 años de edad y una niña de 11 años, quienes consultaron el 8 de diciembre con cuadro clínico consistente en fiebre, escalofrío, sudoración, mialgias, náuseas, cefalea y somnolencia.

El diagnóstico se confirmó mediante el examen de gota gruesa, cuyo resultado inicial fue malaria mixta ( $P$. vivax y $P$. falciparum), aunque después de la revisión de las placas en el Laboratorio Departamental de Salud Pública del Quindío, se determinó que se trataba de malaria por $P$. vivax.

En la entrevista, los menores y sus acudientes manifestaron no tener antecedentes de viaje a zonas endémicas para malaria y llevar siete años de residencia en el sector del parque La Secreta, ubicado en zona de ladera del barrio Arenales, en el municipio de Armenia. Esta zona es fundamentalmente de bosque primario, con algunas áreas intervenidas por el hombre, y termina en una parte plana que bordea la ribera del río Quindío.

\section{Investigación de campo}

Debido a que en el municipio no se habían reportado casos autóctonos de malaria, la Secretaría de Salud de Armenia hizo una investigación de campo antes de notificar oficialmente los casos al Sistema Nacional de Vigilancia en Salud Pública, Sivigila.

En dicha investigación, llevada a cabo el 14 de diciembre de 2012, se capturaron formas larvarias del mosquito Anopheles, las cuales se enviaron al Laboratorio Departamental de Salud Pública del Quindío para su clasificación entomológica.

Se hizo una búsqueda activa de personas con sintomatología compatible con malaria que residieran en el sector donde habitaban los pacientes de los casos confirmados. Se tomaron muestras para el examen de gota gruesa con el fin de hacer el diagnóstico de malaria y otras por venopunción para la determinación de lgM contra el virus del dengue a las personas que cumplían con la definición de caso probable. Algunos pacientes con sintomatología compatible se remitieron al Hospital del Sur en Armenia el 14 de diciembre, y el 17 de diciembre, el personal de laboratorio de Redsalud de Armenia tomó las muestras de los demás en sus domicilios.

\section{Manejo clínico de los pacientes}

Los dos pacientes iniciales recibieron tratamiento para malaria por $P$. vivax no complicada de acuerdo con el protocolo de atención de malaria de 2010 del Ministerio de Salud y Protección Social de Colombia, y según el peso de cada niño: durante el primer día, una dosis de $10 \mathrm{mg} / \mathrm{kg}$ de cloroquina, para la eliminación de esquizontes en sangre, seguida de $7,5 \mathrm{mg} / \mathrm{kg}$ en el segundo día y $7,5 \mathrm{mg} /$ $\mathrm{kg}$ en el tercer día, y una dosis total de $3,5 \mathrm{mg} / \mathrm{kg}$ de primaquina para la eliminación de esquizontes en tejido administrada por vía oral en un esquema de $0,25 \mathrm{mg} / \mathrm{kg}$ al día durante 14 días (14). Se hizo control con nuevo examen de gota gruesa cuatro días después del inicio del tratamiento, así como en los días 7,14, 21 y 28 del tratamiento para verificar la curación parasitológica.

\section{Actividades de investigación entomológica y de control}

Después de recibir la notificación de la presencia de dos casos de malaria, en el Hospital del Sur se aplicó la encuesta epidemiológica a los dos pacientes positivos para malaria, y se ubicó su vivienda para identificar la especie de Anopheles predominante en el sector del parque La Secreta, determinar los 
factores ambientales, físicos y biológicos inherentes al vector que permiten la transmisión de Plasmodium spp. y tomar las medidas de control más adecuadas. Los funcionarios del grupo de zoonosis y vectores de la Secretaría de Salud de Armenia capturaron larvas y adultos para las muestras.

Ante la confirmación de los dos casos y la identificación del vector en sus formas larvaria y adulta, se implementaron las acciones de control social, físico y químico del mosquito vector siguiendo las orientaciones del Instituto Nacional de Salud y del ente departamental.

La Secretaría Municipal de Salud organizó sesiones de educación sanitaria entre los habitantes del sector sobre las medidas de protección personal, como son el uso de ropas adecuadas, es decir, camisas y pantalones de manga larga, y el uso de repelentes y de toldillos (en lo posible impregnados con insecticidas).

Las medidas de control físico consistieron en el empleo de mallas o anjeos para impedir el ingreso del vector a las viviendas, la recolección de recipientes y objetos que pudieran contener agua y servir de potenciales criaderos, el cubrimiento de los tanques o recipientes para el agua de consumo y el drenaje de charcos y de áreas pantanosas cercanas a los márgenes del río.

En los sitios inundables y en las excavaciones realizadas para la cría de peces, se aplicó manualmente el larvicida biológico Vectolex ${ }^{\circledR}$ a base de Bacillus sphaericus, formulación granulada, a razón de 0,15 $\mathrm{g} / \mathrm{m}^{2}$, según las recomendaciones del fabricante (15). Se supervisó la presencia de larvas de mosquitos dos días después de la aplicación del larvicida.

Posteriormente, el 26 de diciembre, se hizo el control químico de mosquitos adultos una vez se informó a todos los habitantes sobre las medidas a tener en cuenta antes, durante y después de la fumigación. Asimismo, se entregó y explicó el documento elaborado por la Secretaría de Salud de Armenia sobre el control de mosquitos.

Se fumigó dentro de las viviendas y en el peridomicilio con equipo rociador X-Pert Hudson ${ }^{\circledR}$ y deltametrina, $50 \mathrm{~g} / \mathrm{L}$ (K-Othrine $50 \mathrm{SC}^{\circledR}$ ) (16), suministrado por la Secretaría Departamental de Salud del Quindío.

\section{Resultados}

\section{Búsqueda activa de casos}

Durante la búsqueda activa en el sector de donde provenían los dos casos índices, nueve de las diez viviendas del sector estaban habitadas; en el censo llevado a cabo se encontraron 50 personas: 33 adultos y 17 menores de edad; 28 personas estaban afiliadas al régimen subsidiado de salud y seis no tenían afiliación alguna. La población residente en el sector estaba dedicada principalmente al cultivo de plátano y café y de huertas caseras, y algunas personas se dedicaban a la extracción de material del río.

Durante la búsqueda activa de casos, se encontraron 11 personas pertenecientes al grupo étnico embera katío, que residían en el municipio hacía un año, aproximadamente, y cumplían con la definición de caso probable y a quienes se les tomaron muestras de sangre para determinar su estado de infección. En los casos en los que el primer examen de gota gruesa dio negativo, se repitió la toma de muestra cada 12 horas hasta las 48 horas, lo que dio como resultado tres personas positivas para $P$. vivax, con parasitemias por debajo de 50.000/ $\mu \mathrm{l}$ (cuadro 1), las cuales recibieron tratamiento según la Guía de Atención Clínica de Malaria, 2010, del Ministerio de Salud y Protección Social (14). Dos de estos tres casos positivos se presentaron en niños que fueron remitidos al Hospital del Sur el 17 de diciembre para valoración por consulta externa e inicio de tratamiento contra la malaria (cuadro 1).

Los resultados de los controles parasitológicos periódicos de los pacientes positivos para hemoparásitos en el examen de gota gruesa fueron todos negativos. En las visitas de seguimiento clínico en busca de signos y síntomas de malaria en los tres pacientes, se los encontró en buenas condiciones generales.

\section{Investigación entomológica}

Descripción del área. El parque La Secreta está situado en el suroriente del municipio de Armenia, el cual se extiende desde el barrio Arenales, con entrada por la vía que conduce al Centro de Zoonosis Municipal y al río Quindío (figura 1). Es una zona de ladera y planicie de inundación, de pendiente regular y alta. Se encuentran nacimientos de aguas, algunos encauzados, que desembocan en el río Quindío. En la planicie se presentan algunas áreas sujetas a inundación por escorrentía y por aguas lluvias retenidas en áreas de depresión a lo largo del margen del río, de difícil drenaje en épocas de invierno y secas en el verano.

El área, declarada de protección en el plan de ordenamiento territorial del municipio de Armenia, fue invadida hace unos 10 años por 12 familias, 
Cuadro1. Información demográfica y resultados del examen de gota gruesa en los casos sospechosos encontrados por búsqueda activa comunitaria en el sector del parque La Secreta, Armenia, Quindío, diciembre de 2012

\begin{tabular}{rlcccc}
\hline No. & Edad & Sexo & Procedencia & Residencia & $\begin{array}{c}\text { Resultado del examen } \\
\text { de gota gruesa }\end{array}$ \\
\hline 1 & 61 años & M & Armenia & Jardínes del Edén & Negativo \\
2 & 49 años & M & Armenia & Parque La Secreta & Negativo \\
3 & 67 años & F & Armenia & Parque La Secreta & Negativo \\
4 & 5 años & M & Santa Cecilia-embera katío & Parque La Secreta & Positivo* $^{*}$ \\
5 & 3 años & F & Santa Cecilia-embera katío & Parque La Secreta & Negitivo* \\
6 & 4 meses & $\mathrm{F}$ & Santa Cecilia-embera katío & Parque La Secreta & Positivo* \\
7 & 7 años & M & Santa Cecilia-embera katío & Parque La Secreta & Negativo \\
8 & 52 años & $\mathrm{F}$ & Armenia & Parque La Secreta & Negativo \\
9 & 59 años & M & Armenia & Parque La Secreta & Negativo \\
10 & 25 años & M & Armenia & Parque La Secreta & Negativo \\
11 & 44 años & M & Armenia & Parque La Secreta & \\
\hline
\end{tabular}

*En los casos positivos en el examen de gota gruesa, se identificaron trofozoítos de P. vivax.

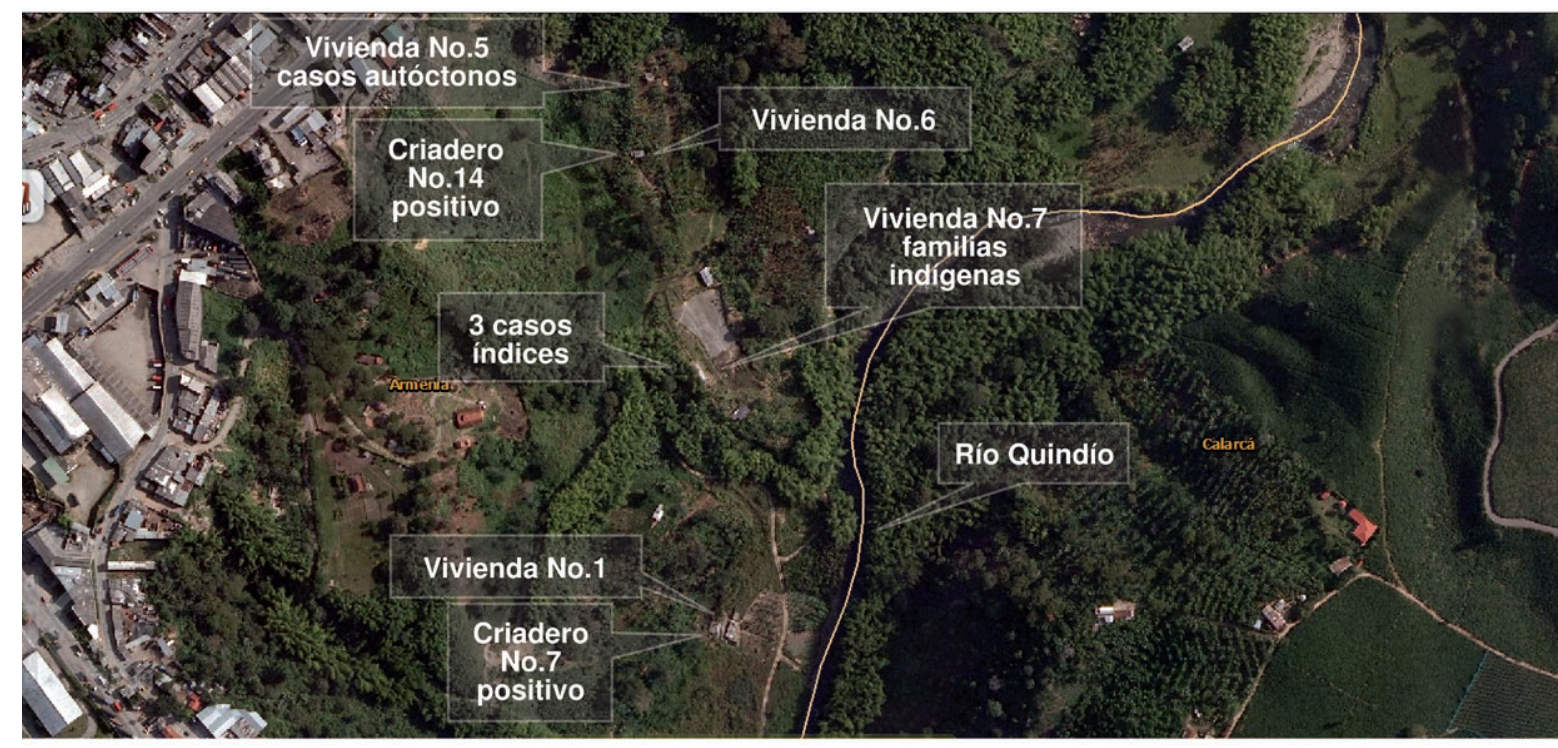

Figura 1. Localización del sector del parque La Secreta, donde se señala la ubicación de los casos índice y de los casos autóctonos de malaria, así como los criaderos de mosquitos, Armenia, Quindío, diciembre de 2012

Vivienda $\mathrm{N}^{\circ} 6$, criadero $\mathrm{N}^{\circ} 1$ (positivo): coordenadas (longitud: $04^{\circ} 31^{\prime} 10,8^{\prime \prime}$; latitud: $\left.75^{\circ} 41^{\prime} 08,2^{\prime \prime}\right)$. Criadero positivo: recipiente artificial, comedero de animales y aves de corral

Vivienda $\mathrm{N}^{\circ} 1$, criadero $\mathrm{N}^{\circ} 7$ (positivo): coordenadas (longitud: 04³0'58,5”; latitud: $\left.75^{\circ} 41^{\prime} 05,1^{\prime \prime}\right)$. Criadero positivo: excavación artificial (dimensiones: $1,0 \mathrm{~m}$ de ancho $\times 0,50 \mathrm{~m}$ de largo $\times 0,40 \mathrm{~m}$ de profundidad) dedicada a cría de peces

en su mayoría de la región. Sin embargo, un año antes se habían establecido tres familias de la comunidad indígena embera katío en una de las viviendas del sector.

De las 10 viviendas encontradas, solo una estaba construida en mampostería, la estructura de las demás estaba conformada por muros y entramados de techo hechos en guadua y madera, cubiertos con teja de zinc, y su área era, en promedio, de 45 $\mathrm{m}^{2}$ construidos. Los pisos de las viviendas estaban cubiertos con esterilla o madera, y algunos eran de tierra. Algunas viviendas solo poseían una o dos habitaciones, no tenían área social y la cocina, con estufa de leña, quedaba en la parte exterior (figuras $2 \mathrm{~A}$ y $\mathrm{B}$ ).

En la zona se observaron algunos suelos boscosos; los demás, ya intervenidos por el hombre, se dedicaban a cultivos permanentes y semipermanentes de café, plátano, yuca, hortalizas y pastos para el ganado y las aves de corral; se encontró un criadero de peces hecho mediante excavaciones cerca del margen del río Quindío. 

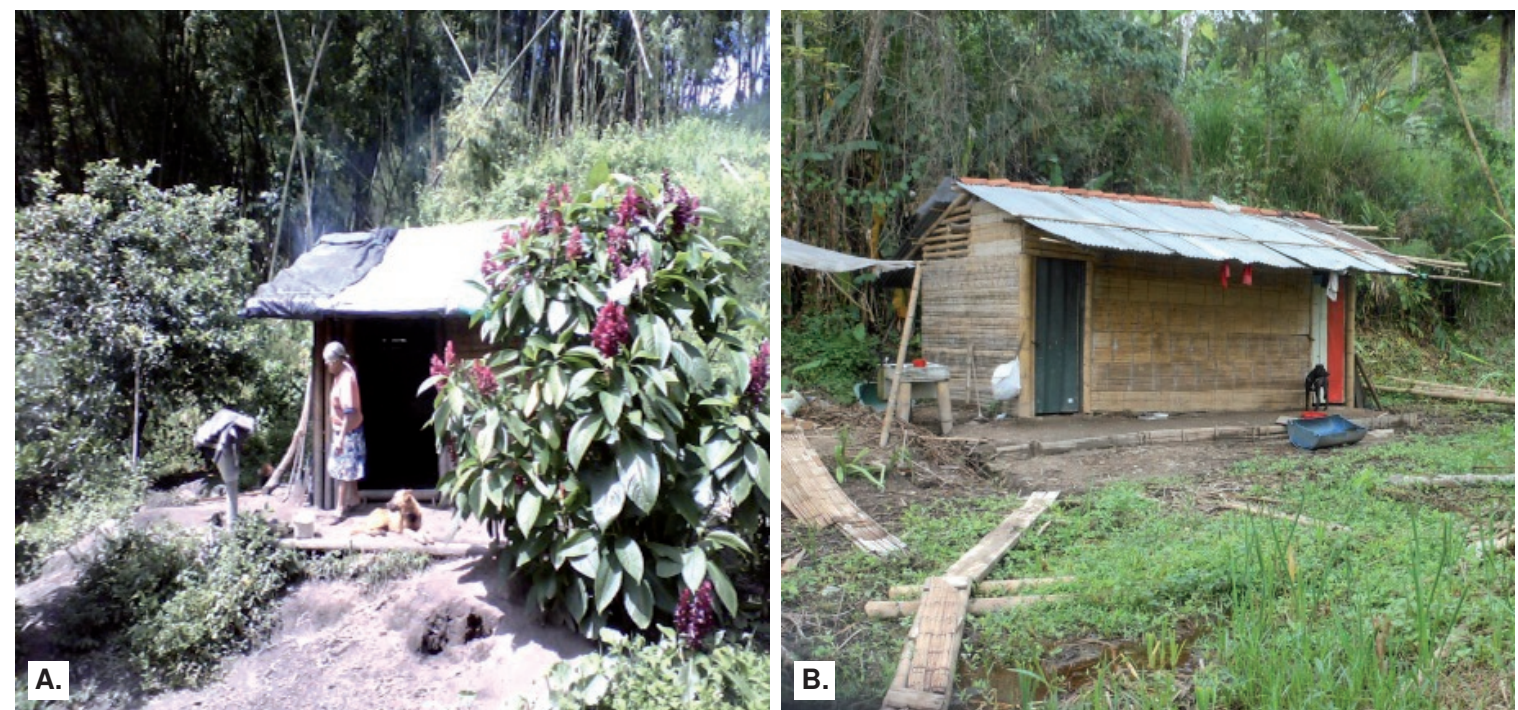

Figura 2. Características de las viviendas del sector del parque La Secreta de Armenia, Quindío, 2012. A. Ambiente del peridomicilio donde se aprecia la cercanía de la vegetación a las casas. B. Panorámica que permite ver los materiales de construcción como guadua, bahareque y zinc.

El agua para el consumo, para la preparación de alimentos y para otros usos domésticos se recolectaba de las lluvias y se almacenaba en diferentes recipientes, desde tanques plásticos hasta canecas improvisadas, algunas cubiertas y otras sin ninguna protección. Los desechos humanos de los inodoros se evacuaban en fosa excavada en el sitio u "hoyo negro". Las viviendas no poseían alcantarillado y las aguas servidas corrían a cielo abierto. En la zona no había servicio de energía. Algunas familias se comunicaban con celular y utilizaban radios de pilas como medio de información. En la mayoría de los casos, la disposición de las basuras se hacía a cielo abierto, y solo algunas personas las enterraban o quemaban.

En el sector vivían de manera permanente 38 personas en nueve casas pertenecientes a 12 familias. La mayoría de los habitantes del área eran nativos de Armenia o de otros municipios del Quindío, a excepción de una persona que vivía desde hacía cinco años en el área y procedía del municipio de Nóvita en el Chocó, y tres familias embera katío. De las 38 personas, 17 eran menores de edad, y de estos, 10 pertenecían a la comunidad indígena. Un total de 17 personas pertenecientes a tres familias eran de la comunidad indígena y convivían en una sola casa. Dos de estas familias residían en el sector desde hacía un año y la tercera familia había llegado tres meses antes. Todos ellos procedían del corregimiento de Santa Cecilia, perteneciente al municipio de Pueblo Rico, departamento de Risaralda, en límites con el Chocó. En dicho municipio la malaria es endémica, y en el 2012 aportó el $82 \%$ de los casos reportados en Risaralda. Según información del DANE, en el 2010 este fue el municipio de Risaralda con el mayor porcentaje de personas en condición de miseria, $31 \%$, en tanto que el $52 \%$ presentaba necesidades básicas insatisfechas (NBI) (17).

Se buscaron los criaderos de anofelinos en toda el área y se elaboró la lista de aquellos positivos para estados inmaduros del vector predominante (figura 1). A5 m de la vivienda de los dos pacientes iniciales, se encontró un recipiente empleado como bebedero de las aves de corral en el cual se recolectaron larvas y pupas de mosquitos del género Anopheles.

El análisis entomológico de las larvas recolectadas en el trabajo de campo se hizo inicialmente en el Laboratorio Departamental de Salud Pública del Quindío, donde los especímenes fueron identificados como An. neomaculipalpus, An. puntimacula y An. calderoni. Para la confirmación de las especies vectoriales, los especímenes fueron remitidos al Laboratorio de Entomología del Instituto Nacional de Salud de Colombia, en donde se reportaron como Anopheles spp., An. punctimacula y Anopheles argyritarsis.

\section{Actividades de control}

El 15 de enero de 2013 se supervisó el área para evaluar las intervenciones básicas realizadas en el parque La Secreta mediante indicadores de proceso, de resultados y de impacto y efectividad. 
Evaluación de los indicadores de proceso. Teniendo en cuenta las recomendaciones de la OMS, se utilizaron parcialmente algunos indicadores para evaluar el proceso operativo de las intervenciones realizadas en el control vectorial de Anopheles spp.

En la evaluación se pudo observar el mejoramiento en el almacenamiento del agua, pues los tanques o canecas ya estaban tapados. Se evidenció el recambio de agua en los bebederos de los animales y la recolección de basuras como tarros, objetos plásticos y de madera. No se encontraron recipientes con formas inmaduras del vector.

Caracterización de criaderos naturales y artificiales. En el sector se encontraron cuatro estanques permanentes excavados artificialmente para la cría de peces (incluido el criadero $\mathrm{N}^{\circ} 7$, descrito como positivo para larvas de Anopheles spp.), que se abastecían con agua de los nacimientos y se ubicaban en la playa, cerca del margen derecho del río Quindío (figura 3). En la investigación de campo inicial, efectuada a mediados del mes de diciembre, se encontraron charcos o áreas anegadas por escorrentía. En el control posterior, entre finales de enero y principios de febrero, estos criaderos se habían secado, pues las lluvias habían cesado, por lo que se los consideró criaderos temporales dependientes de los períodos de lluvias, los cuales se extendieron desde diciembre de 2012 hasta principios de enero de 2013, seguidos de un período de sequía hasta abril de 2013. A mediados de diciembre de 2012 se aplicó manualmente en

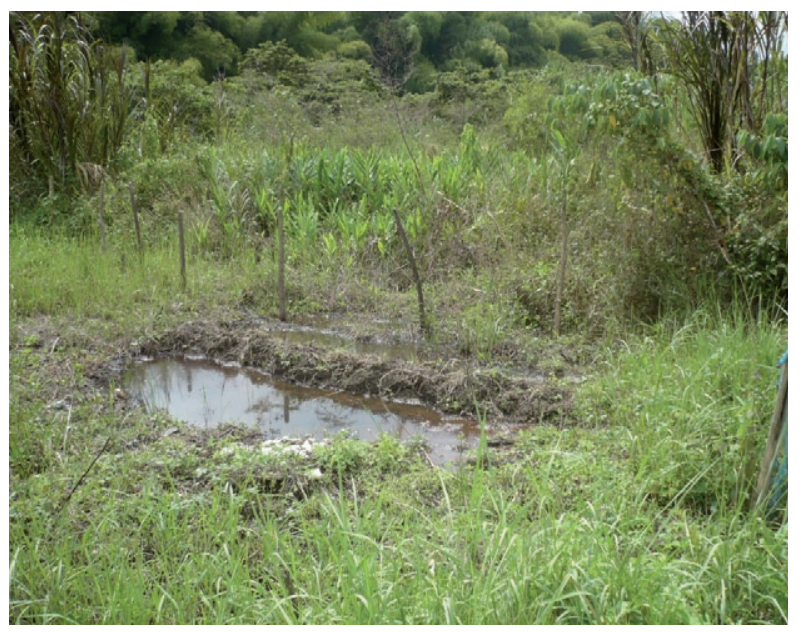

Figura 3. Pozo superficial excavado artificialmente para la cría de peces (dimensiones: 1,0 $\mathrm{m}$ de ancho $\times 0,50 \mathrm{~m}$ de largo $\times 0,40$ $\mathrm{m}$ de profundidad), identificado como criadero $\mathrm{N}^{\circ} 7$ (positivo para formas larvales de Anopheles). Coordenadas (longitud: 04³0'58,5"; latitud: $\left.75^{\circ} 41^{\prime} 05,1^{\prime \prime}\right)$ estos sitios inundables, así como en las excavaciones realizadas para la cría de peces, el larvicida biológico Vectolex ${ }^{\circledR}$, elaborado a base de B. sphaericus (15), como parte de las actividades de control del foco; además, se capturaron larvas dos días y un mes después de la aplicación y no se encontró población larvaria de Anopheles spp. ni de Culex spp.

\section{Discusión}

Según la Secretaría de Salud de Armenia, los casos de malaria en la ciudad son importados, y proceden, en su mayoría, del municipio de Pueblo Rico, Risaralda. Sin embargo, la existencia de criaderos de mosquitos del género Anopheles, como los encontrados en la ribera del río Quindío, permite el establecimiento de un ciclo de transmisión autóctono en sectores de la ciudad donde conviven huéspedes humanos infectados por mosquitos con capacidad vectorial, como en el caso del brote epidémico de malaria autóctona que aquí se reporta, en el cual se evidenció claramente la triada ecológica conformada por el agente etiológico, Plasmodium spp., aportado en este caso por los individuos infectados provenientes de lugares endémicos; por los individuos sensibles, es decir, los niños de la comunidad indígena que fueron los casos índices, y por la vía de transmisión, en este caso los mosquitos vectores Anopheles spp., An. punctimacula y An. argyritarsis, identificados en los criaderos encontrados en la comunidad, así como el medio ambiente que permitió la interacción de estos elementos. Cuando se rompe el equilibrio entre estos tres factores: agente, huésped y medio ambiente, sobreviene la enfermedad, cuya frecuencia puede aumentar o disminuir por dicho efecto (18).

De acuerdo con los datos recolectados en las historias clínicas y en los diarios de campo, se planteó la hipótesis inicial de que la infección pudo haberse propagado a partir de los dos niños diagnosticados con malaria por contacto con los mosquitos en su hábitat natural, los cuales se infectaron por la picadura de personas de la comunidad indígena embera katío procedentes del municipio de Santa Cecilia en límites entre Risaralda y Chocó, algunos llegados al sector hacía aproximadamente un año, pero otros, solo tres meses antes. Vale la pena recordar que la zona de procedencia es un área endémica para malaria.

Si bien Armenia se localiza en la zona andina colombiana y según el índice parasitario anual no se le considera un municipio en el que la malaria 
sea endémica (19), sus condiciones ambientales de humedad, altura sobre el nivel medio del mar $(1.483 \mathrm{msnm})$ y temperatura promedio $\left(18 \mathrm{a} 21^{\circ} \mathrm{C}\right)$ son propicias para el desarrollo del mosquito vector y la transmisión de Plasmodium spp. Sin embargo, los estudios de vectores que permanentemente lleva a cabo la Secretaría de Salud de Armenia como parte del programa de control de la malaria, han permitido identificar numerosos criaderos del mosquito Anopheles en los lugares aledaños al río Quindío. Entre el sector de La María y la vereda Cristales, se ha evidenciado la presencia de vectores desde antes del sismo de 1999 y hasta la fecha, por lo cual la autoridades sanitarias de la ciudad deben estar preparadas para la aparición de casos de malaria autóctona, más si se tiene en cuenta la migración constante de integrantes de las comunidades indígenas procedentes de zonas endémicas para malaria, así como de habitantes del municipio de Armenia que viajan frecuentemente a estas y otras zonas del país y regresan con síntomas de la enfermedad, de manera que deben iniciar o continuar tratamientos. Estas personas habitan, por lo general, en áreas cercanas a la zona urbana, principalmente en los márgenes del río Quindío, donde se han encontrado criaderos permanentes y transitorios de los vectores Anopheles spp., An. punctimacula y An. argyritarsis.

Esta situación podría asemejarse al brote epidémico de malaria descrito por Benítez, et al., en el 2004 en la parroquia Guaramacal del estado Trujillo, Venezuela, área considerada históricamente como libre de malaria y cuya altitud alcanza los 2.200 msnm, en la cual se detectaron nueve casos de malaria asociados con la presencia de cuatro especies del género Anopheles, subgénero Kerteszia, identificados como Anopheles homunculus, An. lepidotus, An. neivai y An. pholidotus, de cuya presencia no existía evidencia anterior en el área de transmisión. Estos autores plantearon, igualmente, que la presencia de casos introducidos se produjo por las frecuentes migraciones de habitantes de otras comunidades donde existían para la época brotes epidémicos de malaria (20).

En relación con las especies de mosquitos encontrados en el brote descrito en este artículo (Anopheles spp., An. punctimacula y An. argyritarsis), hay que considerar que previamente, en el 2001, durante la epidemia de malaria ocurrida después del terremoto de 1999 en el municipio de La Tebaida, Quindío, Olano, et al., reportaron la presencia de Anopheles pseudopunctipennis (Theobald, 1901) y An. punctimacula (Dyar \& Knab, 1906), cuyas formas inmaduras (larvas y pupas), se crían en charcas de agua lluvia, lagunas, remansos de quebradas y depósitos artificiales de agua (tanques bajos a la intemperie y bebederos de animales), así como en huecos y pisadas de animales con agua lluvia acumulada, condiciones todas estas presentes en la comunidad donde se presentó el brote en Armenia (21). Sin embargo, Quiñones, et al., encontraron que en el Putumayo, An. punctimacula no es un vector natural de $P$. vivax (22).

Según Calle, et al., la mayoría de los vectores de los países de la región tropical pertenecen al subgénero Nyssorhynchus, y los principales son: Anopheles (Nyssorhynchus) darling (Root), Anopheles (Nys.) albimanus (Wiedemann) y Anopheles (Nys.) nuneztovari (Gabaldón) (23), aunque en el 2013, Orjuela, et al., señalaron otras especies de este subgénero como posibles transmisores de la enfermedad (24).

En 1993, Rubio-Palis planteó que An. argyritarsis tal vez no era vector de Plasmodiumspp. en Venezuela, en Brasil, ni en otros países latinoamericanos (25). Igualmente, en el 2013, Lardeux, et al., estudiaron la capacidad vectorial de esta especie en los Andes bolivianos y no identificaron parásitos de Plasmodium en este mosquito, lo que les indicó que no era un vector de malaria en los sitios bajo estudio (26). En 1997, Olano, et al., observaron la presencia de formas inmaduras de An. albimanus y An. nuñeztovari en excavaciones de minería, lagos y estanques para piscicultura localizados en la zona periurbana del municipio de Buenaventura (9). Este último hallazgo es similar al del presente estudio, en el cual se encontraron formas inmaduras de anofelinos en estanques para piscicultura. Si bien Parra-Henao, et al., reportaron en el 2011 la presencia de larvas de An. argyritarsis en criaderos artificiales en el cañón del río Sogamoso, Santander, no encontraron circulación de parásitos del género Plasmodium en la zona estudiada (27). En Belém, estado de Pará, Brasil, Silva, et al., describieron la especie entre la variada fauna de anofelinos que encontraron, pero tampoco la implicaron en la transmisión de malaria (28). En el 2010, Dantur, et al., reportaron An. argyritarsis como una de las dos especies de anofelinos más abundantes en las montañas del noroccidente de Argentina, pero señalaron que su papel como vector de malaria era aún desconocido (29).

Por todo lo anterior, es posible que el hallazgo de este mosquito en la comunidad donde ocurrió el brote aquí descrito no estuviera relacionado con 
la transmisión de parásitos, por lo que queda sin resolver la pregunta de cuál fue el vector que transmitió la infección a los individuos sensibles en esta comunidad.

Vale la pena resaltar que la pronta intervención en este brote epidémico de malaria autóctona, consistente en el tratamiento farmacológico apropiado de las personas infectadas, así como en las medidas de control de foco instauradas para eliminar el mosquito y las actividades de educación para la comunidad, permitió su control exitoso y evitó la propagación de la enfermedad al resto de la ciudad de Armenia.

\section{Conflicto de intereses}

Los autores del presente artículo declaramos que no existe ningún conflicto de intereses.

\section{Financiación}

La presente investigación fue financiada por la Secretaría de Salud de Armenia y la Universidad del Quindío.

\section{Referencias}

1. García LS. Malaria. Clin Lab Med. 2010;30:93-129. http:// dx.doi.org/10.1016/j.cll.2009.10.001

2. Centers for Disease Control and Prevention. Malaria facts. Fecha de consulta: 26 de julio de 2013. Disponible en: http://www.cdc.gov/malaria/about/facts.html.

3. Cotter C, Sturrock H, Hsiang M, Liu J, Phillips A, Hwang $\mathrm{J}$, et al. The changing epidemiology of malaria elimination: New strategies for new challenges. Lancet. 2013;382:90011. http://dx.doi.org/10.1016/S0140-6736(13)60310-4

4. Ministerio de Salud y Protección Social, Federación Médica Colombiana. Malaria. Memorias. Fecha de consulta: 10 de septiembre de 2014. Disponible en: http:// www.minsalud.gov.co/sites/rid/Lists/BibliotecaDigital/RIDE/ VS/TH/memorias_malaria.pdf.

5. Instituto Nacional de Salud. Temas de interés. Malaria. Fecha de consulta: 10 de septiembre de 2014. Disponible en: http://www.ins.gov.co/temas-de-interes/Paginas/malaria. aspx.

6. Organización Panamericana de la Salud. Informe de la situación del paludismo en las Américas, 2008. Washington, D.C: Organización Panamericana de la Salud; 2010.

7. Aristizábal D, Mejía I, Pedroza CE, Cárdenas M, Bolaños C, Ballesteros VL, et al. Uso de la inteligencia epidemiológica con participación social para fortalecer la gestión del programa, mejorar el acceso al diagnóstico y tratamiento y ejecutar intervenciones eficaces para la prevención y control de la malaria, 2010 - 2015. Fecha de consulta: julio 26 de 2013. Disponible en: http://www.proyectomalariacolombia. co/files/CajadeHerramientasobj.3/Comparativo\%20Epi\%20 2010-2011-2012\%20PMC.pdf.

8. Botero D, Restrepo M. Parasitosis humana. 5a edición. Medellín: CIB; 2012. p. 215-73.
9. Olano V, Carrasquilla G, Méndez F. Transmisión de la malaria urbana en Buenaventura, Colombia: aspectos entomológicos. Rev Panam Salud Pública. 1997;1:287-94. http://dx.doi.org/10.1590/S1020-49891997000400005

10. Ochoa J, Osorio L. Epidemiología de la malaria urbana en Quibdó, Chocó. Biomédica. 2006;26:278-85. http://dx.doi. org/10.7705/biomedica.v26i2.1417

11. Gobernación del Quindío. Organización del departamento. Fecha de consulta: julio 26 de 2013. Disponible en: http:// www.quindio.gov.co/home/categoria.php?id_item=33\&id_ categoria $=126$.

12. Secretaría de Salud de Armenia. Boletín epidemiológico. Armenia 2008. Armenia: Pixel Publicidad; 2008. p. 12.

13. Instituto Nacional de Salud. Protocolo de vigilancia y control de la malaria. Fecha de consulta: 21 de enero de 2014. Disponible en: http://www.ins.gov.co/lineas-de-accion/ Subdireccion-Vigilancia/sivigila/Protocolos\%20SIVIGILA/ PRO\%20Malaria.pdf.

14. Ministerio de Salud y Protección Social. República de Colombia. Guía de Atención Clínica de Malaria 2010. Fecha de consulta: 10 de septiembre de 2013. Disponible en: http://www.acin.org/acin/new/Portals/0/Templates/guia\%20 de\%20atencion\%20clinica\%20de\%20malaria\%202010.pdf.

15. Valent Bio Sciences Corporation. Vectolex® FG biological larvicide. Fecha de consulta: 26 de mayo de 2013. Disponible en: http://publichealth.valentbiosciences.com/ docs/resources/vectolex-sup-sup-fg---specimen-label.pdf.

16. SANAM. K-OTHRINE SC 50. Fecha de consulta: 25 de mayo de 2014. Disponible en: http://www.sanambiental. com/index.php/productos/linea-bayer/item/k-othrine-sc-50.

17. Sivigila. Malaria en el departamento de Risaralda, 2012. Fecha de consulta: 26 de mayo de 2014. Disponible en: http://www.risaralda.gov.co/site/salud/intradocuments/ webDownload/malaria_960.

18. Toledo-Curbelo G. Fundamentos de salud pública. Segunda edición. La Habana: Editorial Ciencias Médicas; 2005. p. 397, 499.

19. Molina AM. Sistemas de información geográfica para el análisis de la distribución espacial de la malaria en Colombia. Revista EIA. 2008;9:91-111.

20. Benítez J, Rodríguez A, Sojo M, Lobo $\mathbf{H}$, Villegas $\mathbf{C}$, Oviedo L, et al. Descripción de un brote epidémico de malaria de altura en área originalmente sin malaria del estado Trujillo, Venezuela. Bol Malariol Salud Ambient. 2004;44:93-9.

21. Olano VA, Brochero HL, Sáenz R, Quiñones M, Molina J. Mapas preliminares de la distribución de especies de Anopheles vectores de malaria en Colombia. Biomédica. $2001 ; 21: 402-8$.

22. Quiñones ML, Ruiz F, Calle DA, Harbach RE, Erazo HF, Linton YM. Incrimination of Anopheles (Nyssorhynchus) rangeli and An. (Nys.) oswaldoi as natural vectors of Plasmodium vivax in Southern Colombia. Mem Inst Oswaldo Cruz. 2006;101:617-23. http://dx.doi.org/10.1590/S007402762006000600007

23. Calle DA, Quiñones ML, Erazo HF, Jaramillo N. Discriminación por morfometría geométrica de once especies de Anopheles (Nyssorhynchus) presentes en Colombia. 
Biomédica. 2008;28:371-85. http://dx.doi.org/10.7705/ biomedica.v28i3.75

24. Orjuela LI, Herrera M, Erazo H, Quiñones ML. Especies de Anopheles presentes en el departamento del Putumayo y su infección natural con Plasmodium. Biomédica. 2013;33:4252. http://dx.doi.org/10.7705/biomedica.v33i1.619

25. Rubio-Palis Y. Is Anopheles argyritarsis a vector of malaria in the neotropical region? J Am Mosq Control Assoc. 1993;9:470-1.

26. Lardeux F, Aliaga C, Tejerina R, Torrez L. Comparison of transmission parameters between Anopheles argyritarsis and Anopheles pseudopunctipennis in two ecologically different localities of Bolivia. Malar J. 2013;12:282. http:// dx.doi.org/10.1186/1475-2875-12-282
27. Parra-Henao G, Delgado D, Alarcón E. Especies de Anopheles en el Cañón del Río Sogamoso, Santander, Colombia, al inicio de la puesta en marcha del proyecto hidroeléctrico. Bol Malariol Salud Ambient. 2012;52:287-94.

28. Silva A, Fraiha-Neto H, Santos C, Segura M, Amaral J, Gorayed I, et al. Fauna anofélica da cidade de Belém, Pará, Brasil: dados atuais e retrospectivos. Cad Saúde Pública. 2006;22:1575-85. http://dx.doi.org/10.1590/S0102$311 \times 2006000800006$

29. Dantur M, Claps G, Santana M, Zaidenberg M, Almirón W. Abundance patterns of Anopheles pseudopunctipennis and Anopheles argyritarsis in northwestern Argentina. Acta Trop. 2010;115:234-41. http://dx.doi.org/10.1016/j. actatropica.2010.04.003 